\title{
Diagnosis of joint invasion in patients with malignant bone tumors: value and reproducibility of direct and indirect signs on MR imaging
}

\author{
Jannis Bodden ${ }^{1,2}$ (D) - Jan Neumann ${ }^{1} \cdot$ Michael Rasper $^{3} \cdot$ Alexander A. Fingerle $^{1} \cdot$ Carolin Knebel $^{4,5}$. \\ Rüdiger von Eisenhart-Rothe ${ }^{4,5} \cdot$ Katja Specht $^{6,5} \cdot$ Carolin Mogler $^{6,5} \cdot$ Christine Bollwein $^{6} \cdot$ Benedikt J. Schwaiger $^{7}$. \\ Alexandra S. Gersing ${ }^{1,8} \cdot$ Klaus Woertler ${ }^{1,5}$
}

Received: 12 September 2021 / Revised: 15 December 2021 / Accepted: 12 January 2022 / Published online: 8 March 2022

(C) The Author(s) 2022

\begin{abstract}
Objectives To evaluate the performance and reproducibility of MR imaging features in the diagnosis of joint invasion (JI) by malignant bone tumors.

Methods MR images of patients with and without JI ( $n=24$ each), who underwent surgical resection at our institution, were read by three radiologists. Direct (intrasynovial tumor tissue (ITT), intraarticular destruction of cartilage/bone, invasion of capsular/ ligamentous insertions) and indirect (tumor size, signal alterations of epiphyseal/transarticular bone (bone marrow replacement/ edema-like), synovial contrast enhancement, joint effusion) signs of JI were assessed. Odds ratios, sensitivity, specificity, PPV, NPV, and reproducibilities (Cohen's and Fleiss' $\kappa$ ) were calculated for each feature. Moreover, the diagnostic performance of combinations of direct features was assessed.

Results Forty-eight patients ( $28.7 \pm 21.4$ years, 26 men) were evaluated. All readers reliably assessed the presence of JI (sensitivity = 92-100 \%; specificity $=88-100 \%$, respectively). Best predictors for JI were direct visualization of ITT (OR $=186-229, p<0.001)$ and destruction of intraarticular bone $(69-324, p<0.001)$. Direct visualization of ITT was also highly reliable in assessing JI (sensitivity, specificity, PPV, NPV $=92-100 \%)$, with excellent reproducibility $(\kappa=0.83)$. Epiphyseal bone marrow replacement and synovial contrast enhancement were the most sensitive indirect signs, but lacked specificity (29-54\%). By combining direct signs with high specificity, sensitivity was increased $(96 \%)$ and specificity $(100 \%)$ was maintained.

Conclusion JI by malignant bone tumors can reliably be assessed on preoperative MR images with high sensitivity, specificity, and reproducibility. Particularly direct visualization of ITT, destruction of intraarticular bone, and a combination of highly specific direct signs were valuable, while indirect signs were less predictive and specific.

Key Points

- Direct visualization of intrasynovial tumor was the single most sensitive and specific (92-100\%) MR imaging sign of joint invasion.

- Indirect signs of joint invasion, such as joint effusion or synovial enhancement, were less sensitive and specific compared to direct signs.

- A combination of the most specific direct signs of joint invasion showed best results with perfect specificity and PPV (both $100 \%$ ) and excellent sensitivity and NPV (both $96 \%$ ).
\end{abstract}

This work originated from the Department of Diagnostic and

Interventional Radiology, Technical University of Munich.

Jannis Bodden

jannis.bodden@tum.de

1 Department of Diagnostic and Interventional Radiology, Technical University of Munich, Ismaninger Str. 22, 81675 Munich, Germany

2 Department of Radiology and Biomedical Imaging, University of California, San Francisco, 185 Berry St, Lobby 6, Suite 350, San Francisco, CA 94107, USA

3 Department of Radiology, Kantonsspital Muensterlingen, Spitalcampus 1, 8596, Muensterlingen, Switzerland
4 Department of Orthopaedic Surgery, Technical University of Munich, Ismaninger Str. 22, 81675 Munich, Germany

5 Interdisciplinary Musculoskeletal Tumor Center, Technical University of Munich, Ismaninger Str. 22, 81675 Munich, Germany

6 Institute of Pathology, Technical University of Munich, Ismaninger Str. 22, 81675 Munich, Germany

7 Department of Diagnostic and Interventional Neuroradiology, Technical University of Munich, Ismaninger Str. 22, 81675 Munich, Germany

8 Department of Neuroradiology, University Hospital, LMU Munich, 81377 Munich, Germany 
Keywords Bone neoplasms $\cdot$ Neoplasm staging $\cdot$ Osteosarcoma $\cdot$ Chondrosarcoma $\cdot$ Magnetic resonance imaging

$\begin{array}{ll}\text { Abbreviations } \\ \text { 95\% CI } & 95 \% \text { confidence interval } \\ \text { ITT } & \text { Intrasynovial tumor tissue } \\ \text { JI } & \text { Joint invasion } \\ \text { MR } & \text { Magnetic resonance } \\ \text { NPV } & \text { Negative predictive value } \\ \text { OR } & \text { Odds ratio } \\ \text { PPV } & \text { Positive predictive value } \\ \text { TSE } & \text { Turbo spin-echo }\end{array}$

\section{Introduction}

Malignant bone tumors account for approximately $6 \%$ of all cancer cases under the age of 20 years $[1,2]$. The most common types of malignant bone neoplasms affecting young patients are osteosarcomas and Ewing sarcomas, with relative frequencies of $50 \%$ and $40 \%$, respectively. Chondrosarcomas can occur at any age, but $70 \%$ of the affected patients are older than 40 years. While radical resection and amputation were the only available treatment options for these tumors until the 1970s, multimodal therapy, including (neo-)adjuvant chemotherapy, radiation therapy, and surgery, has become the reference standard today [3]. However, neoadjuvant chemotherapy has been found not to impact imaging results or resectability in a favorable manner and resection of the adjacent joint remains inevitable in most cases $[1,4]$. The surgical approach is determined by the joint invasion (JI) status: whereas unaffected joints may be resected intraarticularly, the presence of JI necessitates extraarticular resection to prevent local recurrence, which is associated with a poor prognosis $[5,6]$. However, extra-articular resection is technically demanding and may lead to unfavorable functional results $[7,8]$.

Magnetic resonance (MR) imaging is the standard imaging method for the local staging of malignant bone tumors [9, 10]. Providing excellent soft tissue contrast and three-dimensional anatomic information, extraosseous tumor growth and the proximity to critical structures, like vessels or nerves, can be assessed precisely [11]. Previous studies have also investigated the feasibility of JI assessment on MR images [10, 12-14]. Schima et al investigated epiphyseal involvement, presence of intrasynovial tumor tissue, invasion of the joint capsule, intraarticular destruction of cortical bone or cartilage, invasion of the cruciate ligaments, and joint effusion as MR criteria for tumor invasion of the knee joint [12]. Ozaki et al defined cartilage disruption, acetabular/epiphyseal signal alterations, and marked joint effusion as signs of JI at the hip [13]. However, information on the performance of these imaging features is scarce and reported specificities for MR-based JI assessment differ dramatically [10, 12, 13]. Moreover, previous studies did not analyze the reproducibility of the individual features assessed on MR imaging.

The aim of this study was thus to evaluate the performance and reproducibility of previously described and new MR imaging features in the diagnosis of JI in patients with malignant bone tumors.

\section{Materials and methods}

\section{Cohort}

Approval of the Institutional Review Board has been obtained prior to this study and written informed consent was waived. Pre-treatment MR examinations of patients with malignant bone neoplasms, who had undergone joint resection at our musculoskeletal tumor center between 01/2009 and 12/2019, were obtained from the imaging database. Examinations obtained after chemotherapy were not included for two reasons: First, apparent tumor size reduction under neoadjuvant treatment should not be mistaken as reversal of J, as intraarticular spread may already have occurred. The planning of tumor resection is therefore almost always based on preoperative images with regard to the initial tumor extent. Second, neoadjuvant treatment may induce signal alterations that may be mistaken for tumor tissue and thus be subject to false-positive assessments [4]. To limit the variability of MR protocols, included exams were required to be performed for primary bone tumor screening purposes. Tumor joint adjacency was defined as tumor invasion of either the metaphysis of the joint-forming long bone, the acetabulum, or the glenoid. Cases not presenting one of these features were excluded. Availability of pre-therapeutic scans was obligatory, as imaging features have been shown to be influenced by neoadjuvant therapy [15-17]. Definitive statements on JI status were obtained from pathologic reports of the local Institute of Pathology. Specimens with unclear reports were reviewed by two pathologists (K.S. and C.B.) and JI status was determined.

\section{Image acquisition and evaluation}

MR images were acquired in at least two planes: short tau inversion recovery/fat-saturated intermediate-weighted and pre- and post-contrast $\mathrm{T}_{1}$-weighted sequences were oriented along the longitudinal axis of the articulating long bone(s), whereas $\mathrm{T}_{2^{-}}$ weighed and fat-saturated post-contrast $T_{1}$-weighed sequences were oriented along the short axis. Detailed information on sequence parameters is available as supplementary material. Images were independently reviewed by three radiologists (K.W., A.A.F., and M.R., with 26, 10, and 8 years of experience in musculoskeletal radiology, respectively) 
using picture archiving and communication system (PACS) workstations. Readers were blinded regarding clinical information, surgical outcomes, and histopathological findings. Images were evaluated using a standardized scoring sheet, assessing the following indirect (1.-4.) and direct (5.-7.) signs of J:

1. Epiphyseal/Acetabular signal abnormalities:

- 1.1 Bone marrow replacement (iso-/hypointense to muscle tissue on $\mathrm{T}_{1}$-weighted images)

- 1.2 Edema-like signal intensity (hyperintense to muscle on $\mathrm{T}_{1}$-weighted images and markedly hyperintense on STIR-/intermediate-weighted/ $\mathrm{T}_{2}$-weighted images).

2. Signal abnormalities affecting the transarticular bone (transarticular to primary tumor site):

2.1 Bone marrow replacement (see 1.1.)

2.2 Edema-like signal intensity (see 1.2.)

3. Synovial contrast enhancement

4. Joint effusion

5. Direct visualization of ITT (tumor tissue definitively crossing the synovial membrane toward the joint space, having direct contact to synovial fluid)

6. Intraarticular destruction of:

6.1 Bone (discontinuity of intraarticular cortical bone)

6.2 Cartilage (discontinuity of articular cartilage)

7. Invasion of:

7.1 Cruciate ligament(s) (knee joint)

7.2 Ligamentum teres (hip joint)

7.3 Capsular insertions (all joints)

Readers further measured the largest tumor diameter in centimeter and tumors were divided into two groups (larger than average, smaller than average), for each reader. The overall imaging diagnoses (JI: yes/no), and the subjective certainty of each reader's diagnosis (5-point Likert-scale, 1 : definitely present; 2: likely present; 3 : uncertain; 4: likely not present; 5 : definitely not present), were documented. Image quality was assessed by one reader using a 4-point ordinal scale (poor, moderate, good, excellent). Reader 1 also documented the MR sequence and image orientation most relevant for the diagnosis. Readings were repeated for intra-reader reproducibility measurements (K.W., 90 days between readings).

\section{Combinations of imaging features}

Following the primary analysis of individual imaging features, three dummy variables were created for Reader 1 , based on the following criteria: MaxSens was defined as positive if at least one of all direct imaging features with a good inter-reader reproducibility (Fleiss' $\kappa \geq 0.6$ ) and a good sensitivity ( $\geq 80$ $\%$, in each reader) was achieved. MaxSens was negative if all of the variable-defining imaging features were negative. MaxSpec was positive if at least one direct imaging feature with a good inter-reader reproducibility (Fleiss' $\kappa \geq 0.6$ ) and a good specificity ( $\geq 80 \%$, in each reader) was positive, and negative if all of the defined imaging features were negative. AllDirect was positive if at least one direct sign of JI was positive, and negative if all direct signs of JI were negative.

\section{Statistical analysis}

Data analysis was performed by B.J.S. and J.B. using STATA version 15 software (StataCorp LP). All statistical tests were performed with a two-sided level of significance $(\alpha)$ of 0.05 . Inter-group differences in demographics were compared using Pearson's chi-square test for categorical data (gender, side, site, tumor type) and an independent samples $t$ test for continuous data (age at diagnosis). In a primary analysis, exact logistic regression models were used to determine associations between the observation of individual imaging features and JI status. Moreover, sensitivity, specificity, positive predictive value (PPV), and negative predictive value (NPV) were calculated using crosstabs for each imaging feature and each reader, respectively. Infinity as upper limit of the 95\% CI was noted as "+inf."

In the secondary analysis, sensitivity, specificity, PPV, and NPV of the aforementioned dummy variables (MaxSens, MaxSpec, and AllDirect) were calculated.

Fleiss' $\kappa$ was used to determine inter-reader reliability for repeated image analysis in each imaging feature between readers. Intra-reader reproducibility for repeated measures was calculated using Cohen's $\kappa$.

\section{Results}

\section{Cohort characteristics}

Of 434 patients with malignant bone tumors treated at our institution, 313 were excluded as metaphyseal invasion was absent and/or patients did not undergo resection (Figure 1). Further 68 patients were excluded, because pre-therapeutic MR imaging was unavailable, and five patients were excluded, because the pathologic report lacked a definitive statement on JI and retrospective assessment of the specimens was impossible. We finally included 48 patients (age in years \pm SD: $28.7 \pm 21.4$ years; sex: 26 men, 22 women) with joint adjacent malignant bone tumors in this study (Table 1). JI was more common in older patients and men were affected more frequently (age, no JI: $16.5 \pm 12.0$ vs. JI $40.8 \pm 22.5, p<0.001$; sex, no JI: 14 women, 10 men; JI: 8 women, 16 men, $p=0.08$ ). Most tumors were 
Table 1 Cohort demographics

\begin{tabular}{lllll}
\hline Parameter & & Without joint invasion $(n=24)$ & With joint invasion $(n=24)$ & Total $(n=24)$ \\
\hline Age, mean \pm Standard deviation (years) & $16.5 \pm 12.0$ & $40.8 \pm 22.5$ & $28.7 \pm 21.4$ \\
Sex, $n(\%)$ & Men & $10 / 24(42 \%)$ & $16 / 24(67 \%)$ & $26 / 48(54 \%)$ \\
& Women & $14 / 24(58 \%)$ & $8 / 24(33 \%)$ & $22 / 48(46 \%)$ \\
Tumor site, $n(\%)$ & Shoulder & $4 / 24(17 \%)$ & $3 / 24(12 \%)$ & $7 / 48(15 \%)$ \\
& Hip & $2 / 24(8 \%)$ & $11 / 24(46 \%)$ & $13 / 48(27 \%)$ \\
& Knee & $18 / 24(75 \%)$ & $10 / 24(42 \%)$ & $28 / 48(58 \%)$ \\
Entity, $n(\%)$ & Osteosarcoma & $17 / 24(71 \%)$ & $13 / 24(54 \%)$ & $11 / 48(23 \%)$ \\
& Chondrosarcoma & $3 / 24(12 \%)$ & $8 / 24(33 \%)$ & $4 / 48(8 \%)$ \\
& Ewing sarcoma & $4 / 24(17 \%)$ & 0 & $1 / 48(2 \%)$ \\
& Pleomorphic sarcoma & 0 & $1 / 24(4 \%)$ & $2 / 48(4 \%)$ \\
\hline
\end{tabular}

located at the knee $(n=28)$, exceeding both tumors at hips $(n=$ $13)$ and shoulders $(n=7)$ combined. However, histopathologic findings revealed a higher prevalence of JI in hips (Figure 2) (11/ $13,85 \%)$ compared to knees $(10 / 28,36 \%)$ and shoulders $(3 / 7$, $43 \%)(p=0.006)$. Tumor entities comprised osteosarcomas $(n=$ $30)$, chondrosarcomas $(n=11)$, Ewing sarcomas $(n=4)$, two secondary malignancies, and one pleomorphic sarcoma. JI was most frequently diagnosed in chondrosarcomas, secondary malignancies, and pleomorphic sarcoma (osteosarcoma 43\%; chondrosarcoma $73 \%$; Ewing sarcoma $0 \%$; others $100 \%, p$ $=0.006$ ).

\section{Image analysis}

Reader 1 rated image quality as good or excellent in 42 of 48 cases and as moderate in the remaining six cases. $\mathrm{T}_{2}$-TSE was regarded as the most valuable sequence for determining JI status in most cases $(35 / 48,72.9 \%)$ and the conclusion certainty was
Fig. 1 Flow diagram of study population and patient recruitment

\section{Patients with malignant bone tumors treated by resection at our institution}

$$
(n=434)
$$

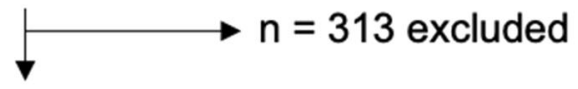

Tumor adjacent to joint

$(n=121)$

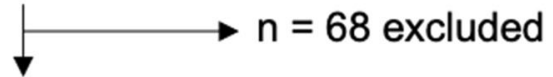

Pre-therapeutic MR imaging performed at our institution $(n=53)$

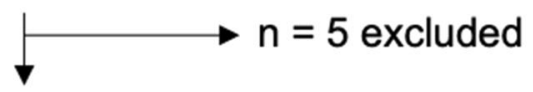

Definitive statement on joint invasion status in pathology reports and/or retrospective assessment of specimen

$$
(n=48)
$$

Patients with joint invasion

$$
(n=24)
$$

Patients without joint invasion $(n=24)$ 
Fig. 2 Chondrosarcoma of the pelvis invading the right hip joint. a Coronal $\mathrm{T}_{1}$-weighted TSE image demonstrates bone marrow replacement at the acetabulum and destruction of intraarticular bone (arrow) caused by bone tumor extending into the pelvis (star). b Axial $\mathrm{T}_{2}$-weighted TSE image shows tumor invasion of the ligamentum teres (arrow) as well as direct visualization of intraarticular tumor tissue in the acetabular fossa (arrowhead). Note the absence of relevant joint effusion and typical lobulated appearance and high signal intensity of cartilaginous mass

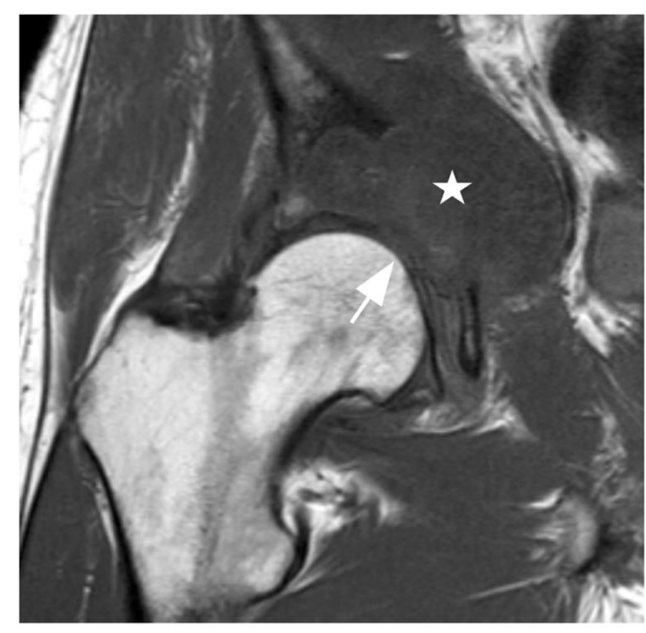

a

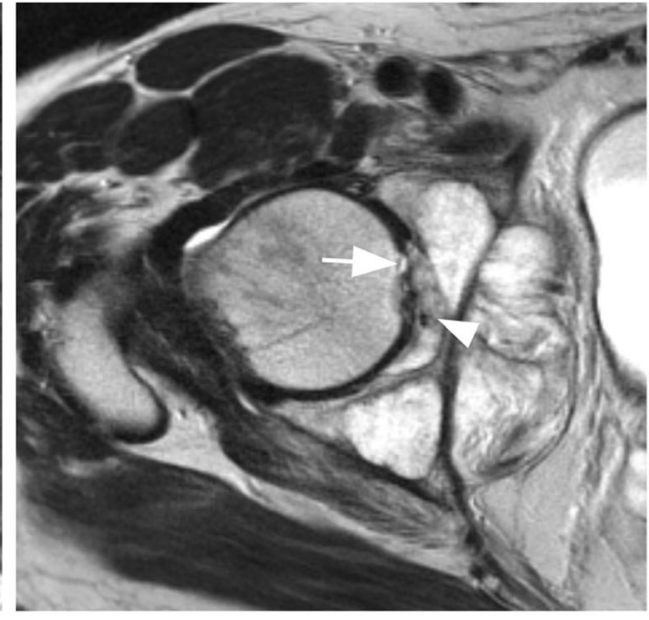

b "uncertain" in none of the cases. Concerning the overall imaging diagnoses, sensitivity and specificity were excellent in all readers (sensitivity: 92-100\%; specificity: 88-100\%) (Table 2).

\section{Indirect signs of joint invasion}

JI was strongly associated with epi-/apophyseal signal alterations reflecting cellular infiltration and findings were highly reproducible (odds ratio $(\mathrm{OR})=15.6-18.3, p=0.002-0.004$; Fleiss $\kappa[95 \% \mathrm{CI}]=0.96[0.80-1.00]$; Table 3$)$. This imaging feature was moreover found to be highly sensitive for JI and had a high NPV in each reader (sensitivity: 96\%, NPV: 9192\%; Table 4). In comparison, edema-like signal alterations at the same location were of lesser diagnostic value $(\mathrm{OR}=0.6-$ $4.8, p=0.03-0.56$; sensitivity: 33-83\%; NPV: 45-75\%). Transarticular bone marrow signal alterations for both cellular infiltration and edema-like signal alterations had an excellent PPV (100\%). However, both of these indirect signs were observed rarely, considerably affecting odds ratios and sensitivities $(\mathrm{OR}=1.0-2.5, p=0.49-1.00$; sensitivity $=1$ to $2 / 24,4$ $8 \%$ ). Joint effusion was associated with increased odds for JI and also showed a substantial inter-reader agreement, but was neither markedly sensitive nor specific and the NPV was limited $(\mathrm{OR}=4.8-25.3, p=<0.001-0.02$; Fleiss $\kappa=0.70$; sensitivity: 54-63\%; specificity: 75-96\%; NPV: 67-69\%). Although odds for JI were comparably higher in cases with synovial contrast enhancement, reproducibility of this imaging feature was only moderate and its specificity limited $(\mathrm{OR}=7.5$ $-8.7, p=0.007-0.06$; Fleiss $\kappa=0.51$; sensitivity: 87-96\%; specificity: $29-54 \%$ ). In our cohort, a maximum tumor diameter greater than $10.5 \mathrm{~cm}$ was not associated with increased odds for JI and this criterion was neither sensitive nor specific $(p \geq$ 0.56; sensitivity: 29-50\%; specificity: 63-79\%).

\section{Direct signs of joint invasion}

All direct signs besides tumor invasion of the ligamentum teres were associated with increased odds for JI (Table 3). Direct visualization of ITT was strongly associated with the histopathological diagnosis of JI (OR [95 \% CI], Reader 1229 [29.8, + inf], $p<0.001$; Reader 2229 [29.8, + inf], $p<0.001$; Reader 3 $186[17.8,10848], p<0.001)$ and associated values for sensitivity, specificity, PPV, and NPV were $\geq 92 \%$ for all readers (Table 4; Figures 3 and 4). Moreover, inter- and intra-reader reproducibility were excellent (Fleiss' $\kappa[95 \% \mathrm{CI}]=0.83[0.67$, 1]; Cohen's $\kappa[95 \% \mathrm{CI}]=0.95[0.86,1.00])$. Odds for JI were high in patients with intraarticular bone destruction $(p<0.001$, respectively), and sensitivity of this sign as well as its NPV both ranged from 96 to $100 \%$, while specificity (71-96\%) and PPV (77-96\%) were reader-dependent (Fleiss' $\kappa$ [95\% CI]; 0.72 $[0.55,0.88])$. Destruction of cartilage showed elevated odds for JI in all readers (Reader 112.5 [1.7, +inf], 0.009; Readers
Table 2 Diagnosis of Joint Invasion: Overall imaging diagnoses

\begin{tabular}{|c|c|c|c|c|c|c|c|c|c|}
\hline \multirow[t]{2}{*}{ Parameter } & \multicolumn{3}{|c|}{ Reader 1} & \multicolumn{3}{|c|}{ Reader 2} & \multicolumn{3}{|c|}{ Reader 3} \\
\hline & $\%$ & & $95 \% \mathrm{CI}$ & $\%$ & & $95 \% \mathrm{CI}$ & $\%$ & & $95 \%$ CI \\
\hline Sensitivity & 92 & $(22 / 24)$ & {$[73,99]$} & 100 & $(24 / 24)$ & {$[86,100]$} & 100 & $(24 / 24)$ & {$[86,100]$} \\
\hline Specificity & 100 & $(24 / 24)$ & {$[86,100]$} & 88 & $(21 / 24)$ & {$[68,97]$} & 88 & $(21 / 24)$ & {$[68,97]$} \\
\hline PPV & 100 & $(22 / 22)$ & {$[85,100]$} & 89 & $(24 / 27)$ & {$[74,96]$} & 89 & $(24 / 27)$ & {$[74,96]$} \\
\hline NPV & 92 & $(24 / 26)$ & {$[76,98]$} & 100 & $(21 / 21)$ & {$[85,100]$} & 100 & $(21 / 21)$ & {$[85,100]$} \\
\hline
\end{tabular}

$P P V$, positive predictive value; $N P V$, negative predictive value; $95 \% C I, 95 \%$ Confidence interval 
Table 3 Associations of imaging features and JI

\begin{tabular}{|c|c|c|c|c|c|c|c|c|c|}
\hline \multicolumn{2}{|l|}{ Imaging feature } & Reader & OR & $95 \% \mathrm{CI}$ & $p^{*}$ & Fleiss' $\kappa$ & $95 \% \mathrm{CI}$ & Cohen's $\kappa$ & $95 \% \mathrm{CI}$ \\
\hline \multirow{3}{*}{\multicolumn{2}{|c|}{ Tumor size $>10.5 \mathrm{~cm}$}} & 1 & 1.0 & {$[0.3,3.8]$} & 1.00 & 0.67 & {$[0.50,0.83]$} & 0.95 & {$[0.86,1.00]$} \\
\hline & & 2 & 1.6 & {$[0.4,7.5]$} & 0.74 & & & & \\
\hline & & 3 & 1.7 & {$[0.5,6.2]$} & 0.56 & & & & \\
\hline \multirow[t]{6}{*}{ Epiphyseal/acetabular } & Bone marrow replacement & 1 & 15.6 & {$[1.9,743]$} & 0.004 & 0.96 & {$[0.80,1.00]$} & 1.00 & {$[1.00,1.00]$} \\
\hline & & 2 & 18.3 & {$[2.2,873]$} & 0.002 & & & & \\
\hline & & 3 & 18.3 & {$[2.2,873]$} & 0.002 & & & & \\
\hline & Edema-like signal intensity & 1 & 0.6 & {$[0.2,2.2]$} & 0.56 & 0.40 & {$[0.24,0.56]$} & 0.52 & {$[0.27,0.77]$} \\
\hline & & 2 & 2.7 & {$[0.7,12.3]$} & 0.21 & & & & \\
\hline & & 3 & 4.8 & {$[1.1,25.4]$} & 0.03 & & & & \\
\hline \multirow[t]{6}{*}{ Transarticular } & Bone marrow replacement & 1 & 1.0 & {$[0.3,+\mathrm{inf}]$} & 1.00 & 0.74 & {$[0.58,0.91]$} & 1.00 & {$[1.00,1.00]$} \\
\hline & & 2 & 1.0 & {$[0.3,+\mathrm{inf}]$} & 1.00 & & & & \\
\hline & & 3 & 2.5 & {$[0.2,+\mathrm{inf}]$} & 0.49 & & & & \\
\hline & Edema-like signal intensity & 1 & 2.5 & {$[0.2,+\mathrm{inf}]$} & 0.49 & 0.74 & {$[0.58,0.91]$} & 1.00 & {$[1.00,1.00]$} \\
\hline & & 2 & 1.0 & {$[0.0,+\mathrm{inf}]$} & 1.00 & & & & \\
\hline & & 3 & 1.0 & {$[0.0,+\mathrm{inf}]$} & 1.00 & & & & \\
\hline \multirow{3}{*}{\multicolumn{2}{|c|}{ Synovial contrast enhancement }} & 1 & 8.7 & {$[1.0,426]$} & 0.06 & 0.51 & {$[0.35,0.68]$} & 0.81 & {$[0.55,1.00]$} \\
\hline & & 2 & 7.5 & {$[1.6,50.1]$} & 0.007 & & & & \\
\hline & & 3 & 7.5 & {$[1.6,50.1]$} & 0.007 & & & & \\
\hline \multirow{3}{*}{\multicolumn{2}{|c|}{ Joint effusion }} & 1 & 4.8 & {$[1.3,21.0]$} & 0.02 & 0.70 & {$[0.53,0.86]$} & 0.77 & {$[0.58,0.96]$} \\
\hline & & 2 & 25.3 & {$[3.1,1204]$} & $<0.001$ & & & & \\
\hline & & 3 & 14.4 & {$[2.6,155]$} & $<0.001$ & & & & \\
\hline \multirow{3}{*}{\multicolumn{2}{|c|}{ Direct visualization of ITT }} & 1 & 229 & {$[29.8,+\mathrm{inf}]$} & $<0.001$ & 0.83 & {$[0.67,1.00]$} & 0.95 & {$[0.86,1.00]$} \\
\hline & & 2 & 229 & {$[29.8,+\mathrm{inf}]$} & $<0.001$ & & & & \\
\hline & & 3 & 186 & {$[17.8,10848]$} & $<0.001$ & & & & \\
\hline \multirow[t]{6}{*}{ Destruction of } & Bone & 1 & 324 & {$[25.2,22596]$} & $<0.001$ & 0.72 & {$[0.55,0.88]$} & 0.91 & {$[0.79,1.00]$} \\
\hline & & 2 & 75.4 & {$[8.7,3780]$} & $<0.001$ & & & & \\
\hline & & 3 & 68.8 & {$[10.0,+\mathrm{inf}]$} & $<0.001$ & & & & \\
\hline & Cartilage & 1 & 12.5 & {$[1.7,+\mathrm{inf}]$} & 0.009 & 0.57 & {$[0.40,0.73]$} & 0.85 & {$[0.65,1.00]$} \\
\hline & & 2 & 37.0 & {$[6.2,430]$} & $<0.001$ & & & & \\
\hline & & 3 & 37.0 & {$[6.2,430]$} & $<0.001$ & & & & \\
\hline \multirow[t]{9}{*}{ Invasion of } & Capsular insertion & 1 & 25.5 & {$[3.7,+$ inf $]$} & $<0.001$ & 0.62 & {$[0.45,0.78]$} & 0.94 & {$[0.82,1.00]$} \\
\hline & & 2 & 9.3 & {$[2.0,62.0]$} & 0.002 & & & & \\
\hline & & 3 & 6.7 & {$[1.6,35.6]$} & 0.007 & & & & \\
\hline & Cruciate ligament & 1 & 61.0 & {$[7.2,+\mathrm{inf}]$} & $<0.001$ & 0.77 & {$[0.55,0.98]$} & 0.81 & {$[0.56,1.00]$} \\
\hline & & 2 & 10.7 & {$[1.3,148]$} & 0.02 & & & & \\
\hline & & 3 & 10.7 & {$[1.3,148]$} & 0.02 & & & & \\
\hline & Ligamentum teres & 1 & 2.3 & {$[0.2,+\mathrm{inf}]$} & 0.54 & 0.79 & {$[0.48,1.00]$} & 1.00 & {$[1.00,1.00]$} \\
\hline & & 2 & 4.6 & {$[0.3,+\mathrm{inf}]$} & 0.26 & & & & \\
\hline & & 3 & 4.6 & {$[0.3,+\mathrm{inf}]$} & 0.26 & & & & \\
\hline
\end{tabular}

* Significant $p$ values are printed bold. OR, odds radio; 95\% CI, 95\% confidence interval; ITT, intrasynovial tumor tissue; + inf, upper limit of $95 \%$ confidence interval was infinite

$2+337.0[6.2,430], p<0.001)$ and a specificity of 92 to $100 \%$. With regard to intraarticular ligaments, analyses demonstrated increased odds for JI in cases affecting the cruciate ligaments, but not the ligamentum teres $(p \leq 0.02$ and $p \geq 0.26$, respectively). Capsular invasion predicted the histopathologic diagnosis of JI in all readers ( $p \leq 0.007$, respectively) and specificity was $\geq$ $83 \%$, for each reader (Figure 5).

\section{Combinations of imaging features}

Direct visualization of ITT and intraarticular destruction of bone met the criteria to be included in the MaxSens variable and MaxSpec comprised direct visualization of ITT, invasion of the capsular insertion, and cruciate ligaments or ligamentum teres. AllDirect had a sensitivity, specificity, 
Table 4 Performance of the analyzed imaging features in diagnosing JI

\begin{tabular}{|c|c|c|c|c|c|c|c|c|c|c|c|c|c|}
\hline \multirow{2}{*}{\multicolumn{2}{|c|}{$\begin{array}{l}\text { Imaging feature } \\
\text { Tumor size }>10.5 \mathrm{~cm}\end{array}$}} & \multirow{2}{*}{$\begin{array}{l}\text { Reader } \\
1\end{array}$} & \multicolumn{3}{|c|}{$\begin{array}{l}\text { Sensitivity } \\
(\%, \mathrm{n},[95 \% \mathrm{CI}])\end{array}$} & \multicolumn{3}{|c|}{$\begin{array}{l}\text { Specificity } \\
(\%, \mathrm{n},[95 \% \mathrm{CI}])\end{array}$} & \multicolumn{2}{|c|}{$\operatorname{PPV}(\%, \mathrm{n}, 95 \% \mathrm{CI})$} & \multicolumn{3}{|c|}{$\operatorname{NPV}(\%, \mathrm{n}, 95 \% \mathrm{CI}])$} \\
\hline & & & 38 & $(9 / 24)$ & {$[18,59)$} & 63 & $(15 / 24)$ & {$[41,81]$} & $50 \quad(9 / 18)$ & {$[32,67]$} & 50 & $(15 / 30)$ & {$[39,61]$} \\
\hline & & 2 & 29 & $(7 / 24)$ & {$[13,51]$} & 79 & $(19 / 24)$ & {$[58,93]$} & $58 \quad(7 / 12)$ & {$[34,79]$} & 53 & $(19 / 36)$ & {$[45,61]$} \\
\hline & & 3 & 50 & $(12 / 24)$ & {$[31,69]$} & 63 & $(15 / 24)$ & {$[43,79]$} & $57 \quad(12 / 21)$ & {$[37,72]$} & 56 & $(15 / 27)$ & {$[37,72]$} \\
\hline \multirow[t]{6}{*}{ Epiphyseal/acetabular } & \multirow{3}{*}{$\begin{array}{l}\text { Bone marrow } \\
\text { replacement }\end{array}$} & 1 & 96 & $(23 / 24)$ & {$[80,100]$} & 42 & $(10 / 24)$ & {$[22,63]$} & $62(23 / 37)$ & {$[54,70]$} & 91 & $(10 / 11)$ & {$[58,99]$} \\
\hline & & 2 & 96 & $(23 / 24)$ & $\begin{array}{l}{[80,} \\
100]\end{array}$ & 46 & $(11 / 24)$ & {$[26,67]$} & $64(23 / 26)$ & {$[55,72]$} & 92 & $(11 / 12)$ & {$[65,99]$} \\
\hline & & 3 & 96 & $(23 / 24)$ & {$[80,99]$} & 46 & $(11 / 24)$ & {$[28,65]$} & $64(23 / 36)$ & {$[48,78]$} & 92 & $(11 / 12)$ & {$[65,99]$} \\
\hline & \multirow{3}{*}{$\begin{array}{l}\text { Edema-like } \\
\text { signal } \\
\text { intensity }\end{array}$} & 1 & 33 & $(8 / 24)$ & {$[16,55]$} & 54 & $(13 / 24)$ & {$[33,74]$} & $42 \quad(8 / 19)$ & {$[26,60]$} & 45 & $(13 / 29)$ & {$[34,56]$} \\
\hline & & 2 & 80 & $(19 / 24)$ & {$[58,93]$} & 42 & $(11 / 24)$ & {$[22,63]$} & $58 \quad(19 / 33)$ & {$[48,67]$} & 67 & $(10 / 15)$ & {$[45,83 \mid$} \\
\hline & & 3 & 83 & $(20 / 24)$ & {$[64,93]$} & 50 & $(12 / 24)$ & {$[31,69]$} & $63 \quad(20 / 32)$ & {$[45,77]$} & 75 & $(12 / 16)$ & {$[51,90]$} \\
\hline \multirow[t]{6}{*}{ Transarticular } & \multirow[t]{3}{*}{$\begin{array}{l}\text { Bone marrow } \\
\text { replacement }\end{array}$} & 1 & 4 & $(1 / 24)$ & {$[0,21]$} & 100 & $(24 / 24)$ & {$[85,100]$} & $100(1 / 1)$ & $\begin{array}{l}{[21,} \\
100]\end{array}$ & 51 & $(24 / 47)$ & {$[39,53]$} \\
\hline & & 2 & 4 & $(1 / 24)$ & {$[0,21]$} & 100 & $(24 / 24)$ & {$[85,100]$} & $100(1 / 1)$ & $\begin{array}{l}{[21,} \\
100]\end{array}$ & 51 & $(24 / 47)$ & {$[39,53]$} \\
\hline & & 3 & 8 & $(2 / 24)$ & {$[1,27]$} & 100 & $(24 / 24)$ & {$[86,100]$} & $100(2 / 2)$ & $\begin{array}{l}{[34,} \\
100]\end{array}$ & 52 & $(24 / 46)$ & {$[49,55]$} \\
\hline & \multirow{3}{*}{$\begin{array}{l}\text { Edema-like } \\
\quad \text { signal } \\
\text { intensity }\end{array}$} & 1 & 8 & $(2 / 24)$ & {$[1,27]$} & 100 & $(24 / 24)$ & {$[86,100]$} & $100(2 / 2)$ & $\begin{array}{c}{[34,} \\
100]\end{array}$ & 52 & $(24 / 46)$ & {$[49,55]$} \\
\hline & & 2 & 4 & $(1 / 24)$ & {$[0,21]$} & 100 & $(24 / 24)$ & {$[85,100]$} & $100(1 / 1)$ & $\begin{array}{l}{[21,} \\
100]\end{array}$ & 51 & $(24 / 47)$ & {$[39,53]$} \\
\hline & & 3 & 4 & $(1 / 24)$ & {$[0,21]$} & 100 & $(24 / 24)$ & {$[85,100]$} & $100(1 / 1)$ & $\begin{array}{l}{[21,} \\
100]\end{array}$ & 51 & $(24 / 47)$ & {$[39,53]$} \\
\hline \multirow{3}{*}{\multicolumn{2}{|c|}{ Synovial contrast enhancement }} & 1 & 96 & $(22 / 23)$ & $\begin{array}{l}{[78,} \\
100]\end{array}$ & 29 & $(7 / 24)$ & {$[12,51]$} & $56 \quad(22 / 39)$ & {$[50,63]$} & 88 & $(7 / 8)$ & {$[48,98]$} \\
\hline & & 2 & 87 & $(20 / 23)$ & {$[66,97]$} & 54 & $(13 / 24)$ & {$[33,74]$} & $65(20 / 31)$ & {$[53,74]$} & 81 & $(13 / 16)$ & {$[59,93]$} \\
\hline & & 3 & 87 & $(20 / 23)$ & {$[66,97]$} & 54 & $(13 / 24)$ & {$[33,74]$} & $65(20 / 31)$ & {$[53,74]$} & 81 & $(13 / 16)$ & {$[59,93]$} \\
\hline \multirow{3}{*}{\multicolumn{2}{|c|}{ Joint effusion }} & 1 & 63 & $(15 / 24)$ & {$[41,81]$} & 75 & $(18 / 24)$ & {$[44,81]$} & $71 \quad(15 / 21)$ & {$[53,84]$} & 67 & $(18 / 27)$ & {$[53,81]$} \\
\hline & & 2 & 54 & $(13 / 24)$ & {$[33,74]$} & 96 & $(23 / 24)$ & {$[79,100]$} & $93(13 / 14)$ & {$[65,99]$} & 68 & $(23 / 34)$ & {$[57,77]$} \\
\hline & & 3 & 58 & $(14 / 24)$ & {$[39,76]$} & 92 & $(22 / 24)$ & {$[74,98]$} & $88 \quad(14 / 16)$ & {$[64,97]$} & 69 & $(22 / 32)$ & {$[51,82]$} \\
\hline \multirow{3}{*}{\multicolumn{2}{|c|}{ Direct visualization of ITT }} & 1 & 92 & $(22 / 24)$ & {$[73,99]$} & 100 & $(24 / 24)$ & {$[86,100]$} & $100(22 / 22)$ & $\begin{array}{l}{[85,} \\
100]\end{array}$ & 92 & $(24 / 26)$ & {$[76,98]$} \\
\hline & & 2 & 100 & $(24 / 24)$ & $\begin{array}{l}{[86,} \\
100]\end{array}$ & 92 & $(22 / 24)$ & {$[73,99]$} & $92(24 / 26)$ & {$[76,98]$} & 100 & $(22 / 22)$ & $\begin{array}{l}{[85,} \\
100]\end{array}$ \\
\hline & & 3 & 96 & $(23 / 24)$ & $\begin{array}{l}{[80,} \\
100]\end{array}$ & 92 & $(22 / 24)$ & {$[74,98]$} & $92 \quad(23 / 25)$ & {$[75,98]$} & 96 & $(22 / 23)$ & {$[80,99]$} \\
\hline \multirow[t]{6}{*}{ Destruction of } & \multirow[t]{3}{*}{ Bone } & 1 & 96 & $(23 / 24)$ & $\begin{array}{l}{[80,} \\
100]\end{array}$ & 96 & $(23 / 24)$ & {$[79,100]$} & $96 \quad(23 / 24)$ & {$[77,99]$} & 96 & $(23 / 24)$ & {$[77,99]$} \\
\hline & & 2 & 96 & $(23 / 24)$ & $\begin{array}{c}{[80,} \\
100]\end{array}$ & 79 & $(19 / 24)$ & {$[56,93]$} & $82 \quad(23 / 28)$ & {$[68,91]$} & 95 & $(19 / 20)$ & {$[73,99]$} \\
\hline & & 3 & 100 & $(24 / 24)$ & $\begin{array}{l}{[86,} \\
100]\end{array}$ & 71 & $(17 / 24)$ & {$[51,85]$} & $77 \quad(24 / 31)$ & {$[60,89]$} & 100 & $(17 / 17)$ & $\begin{array}{l}{[82,} \\
100]\end{array}$ \\
\hline & \multirow[t]{3}{*}{ Cartilage } & 1 & 29 & $(7 / 24)$ & {$[13,51]$} & 100 & $(24 / 24)$ & {$[86,100]$} & $100(7 / 7)$ & $\begin{array}{l}{[65,} \\
100]\end{array}$ & 59 & $(24 / 41)$ & {$[52,65]$} \\
\hline & & 2 & 79 & $(19 / 24)$ & {$[58,93]$} & 92 & $(22 / 24)$ & {$[73,99]$} & $90 \quad(19 / 21)$ & {$[71,97]$} & 81 & $(22 / 27)$ & {$[67,91]$} \\
\hline & & 3 & 79 & $(19 / 24)$ & {$[58,93]$} & 92 & $(22 / 24)$ & {$[73,99]$} & $90 \quad(19 / 21)$ & {$[71,97]$} & 81 & $(22 / 27)$ & {$[67,91]$} \\
\hline \multirow[t]{7}{*}{ Invasion of } & \multirow[t]{3}{*}{$\begin{array}{l}\text { Capsular } \\
\text { insertion }\end{array}$} & 1 & 46 & $(11 / 24)$ & {$[28,65]$} & 100 & $(24 / 24)$ & {$[86,100]$} & $100(11 / 11)$ & $\begin{array}{l}{[74,} \\
100]\end{array}$ & 65 & $(24 / 37)$ & {$[49,78]$} \\
\hline & & 2 & 58 & $(14 / 24)$ & {$[37,78]$} & 88 & $(21 / 24)$ & {$[68,97]$} & $82 \quad(14 / 17)$ & {$[61,93]$} & 68 & $(21 / 31)$ & {$[56,78]$} \\
\hline & & 3 & 58 & $(14 / 24)$ & {$[37,78]$} & 83 & $(20 / 24)$ & {$[39,76]$} & $78 \quad(14 / 18)$ & {$[55,91]$} & 67 & $(20 / 30)$ & {$[49,81]$} \\
\hline & \multirow[t]{3}{*}{$\begin{array}{l}\text { Cruciate } \\
\text { ligament }\end{array}$} & 1 & 80 & $(8 / 10)$ & {$[44,97]$} & 100 & $(18 / 18)$ & {$[81,100]$} & $100(8 / 8)$ & $\begin{array}{l}{[68,} \\
100]\end{array}$ & 90 & $(18 / 20)$ & {$[72,97]$} \\
\hline & & 2 & 60 & $(6 / 10)$ & {$[26,88]$} & 89 & $(16 / 18)$ & {$[65,99]$} & $75 \quad(6 / 8)$ & {$[41,93]$} & 80 & $(16 / 20)$ & {$[65,90]$} \\
\hline & & 3 & 60 & $(6 / 10)$ & {$[26,88]$} & 89 & $(16 / 18)$ & {$[65,99]$} & $75 \quad(6 / 8)$ & {$[41,93]$} & 80 & $(16 / 20)$ & {$[65,90]$} \\
\hline & $\begin{array}{l}\text { Ligamentum } \\
\text { teres }\end{array}$ & 1 & 55 & $(5 / 11)$ & {$[23,83]$} & 100 & $(2 / 2)$ & {$[16,100]$} & $100(6 / 6)$ & $\begin{array}{l}{[34,} \\
100]\end{array}$ & 29 & $(2 / 7)$ & {$[17,43]$} \\
\hline
\end{tabular}


Table 4 (continued)

\begin{tabular}{|c|c|c|c|c|c|c|c|c|c|c|}
\hline \multirow[t]{3}{*}{ Imaging feature } & \multirow{2}{*}{$\begin{array}{l}\text { Reader } \\
2\end{array}$} & \multicolumn{2}{|c|}{$\begin{array}{l}\text { Sensitivity } \\
(\%, \mathrm{n},[95 \% \mathrm{CI}])\end{array}$} & \multicolumn{2}{|c|}{$\begin{array}{l}\text { Specificity } \\
(\%, \mathrm{n},[95 \% \mathrm{CI}])\end{array}$} & \multicolumn{2}{|c|}{$\operatorname{PPV}(\%, \mathrm{n}, 95 \% \mathrm{CI})$} & \multicolumn{3}{|c|}{$\operatorname{NPV}(\%, \mathrm{n}, 95 \% \mathrm{CI}])$} \\
\hline & & $73 \quad(8 / 11)$ & {$[39,94]$} & $100(2 / 2)$ & {$[16,100]$} & $100(8 / 8)$ & $\begin{array}{l}{[68,} \\
100]\end{array}$ & 40 & $(2 / 5)$ & {$[20,64]$} \\
\hline & 3 & $73 \quad(8 / 11)$ & {$[39,94]$} & $100(2 / 2)$ & {$[16,100]$} & $100(8 / 8)$ & $\begin{array}{l}{[68,} \\
100]\end{array}$ & 40 & $(2 / 5)$ & {$[20,64]$} \\
\hline
\end{tabular}

$P P V$, positive predictive value; $N P V$, negative predictive value; $95 \% C I, 95 \%$ confidence interval; ITT, intrasynovial tumor tissue

PPV, and NPV of $96 \%$ with one false-positive and one falsenegative case (95\% CI; sensitivity, specificity: 79, 100; PPV, NPV: 77, 99). While results for MaxSens were the same, MaxSpec had a specificity and PPV of $100 \%$ [86, 100], a sensitivity of $96 \%[79,100]$, and a NPV of $96 \%[78,99]$, with only one false-negative case.

\section{Discussion}

This study evaluated the diagnostic performance and reproducibility of MR imaging features in the detection of JI by malignant bone tumors. Similar to previous population-based studies, osteosarcomas were the most frequent entity and the knee was the most common location for malignant bone neoplasms in our cohort [1]. Therefore, despite the relatively low number of included cases, we regard the external validity of our cohort as substantial.

Correct assessment of JI status in preoperative MR examinations is of great importance to orthopedic surgeons, as falsepositive diagnoses lead to unnecessary extensive resections with high complication rates and worse functional outcomes, while false-negative diagnoses may increase the risk of local recurrence due to intra-operative spread of malignant cells
Fig. 3 Osteosarcoma of the left distal femur invading the knee joint. a Sagittal $\mathrm{T}_{1}$-weighted TSE image shows metaphyseal mass (star) with epiphyseal extension and posterior soft tissue component. b Corresponding post-contrast image reveals inhomogeneous enhancement of the bone lesion as well as synovial enhancement at the posterior joint recess (arrows). c Sagittal intermediate-weighted TSE image with fat suppression and $\mathbf{d}$ axial $\mathrm{T}_{2}$-weighted TSE image show JI via the posterior capsular insertion and directly visualize intraarticular tumor tissue in contact with joint fluid and cartilage surface of the lateral femoral condyle (arrows)
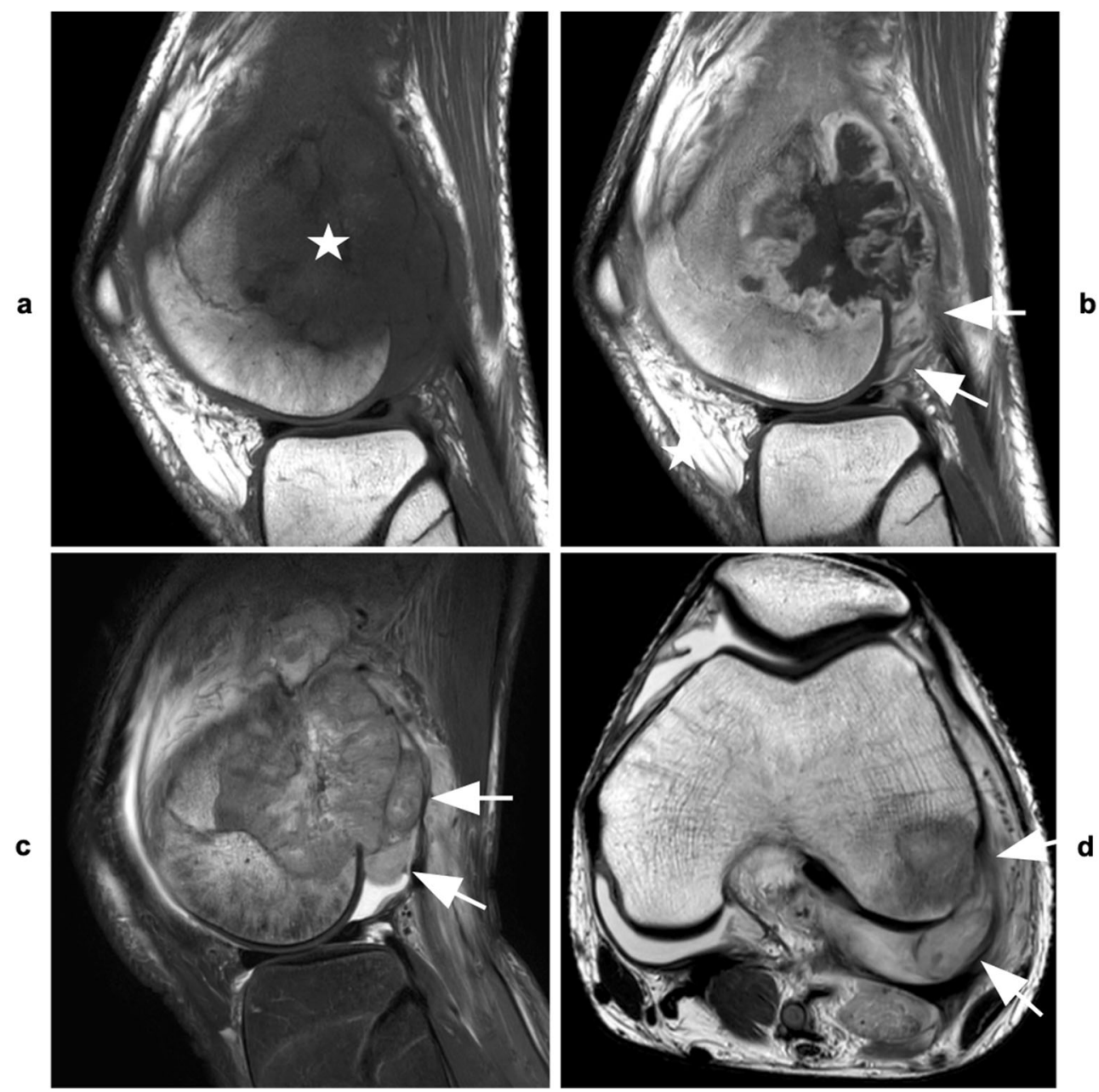
Fig. 4 Chondrosarcoma of the right distal femur invading the knee joint at two different sites. a Sagittal $\mathrm{T}_{1}$-weighted and $\mathbf{b}$ corresponding intermediateweighted TSE images with fat suppression demonstrate bone marrow replacement in the metaphysis and epiphysis of the distal femur (stars), destruction of intraarticular bone at the roof of the intercondylar notch (arrows), and tumor invasion of the anterior cruciate ligament (arrowheads). c, $\mathbf{d}$ Consecutive axial $\mathrm{T}_{2-}$ weighted images show destruction of bone and articular cartilage at the femoral trochlea (arrows) as well as tumor nodules in contact with joint fluid and cartilage surface of the patella (arrowheads) indicating invasion of the femoro-patellar joint compartment

a
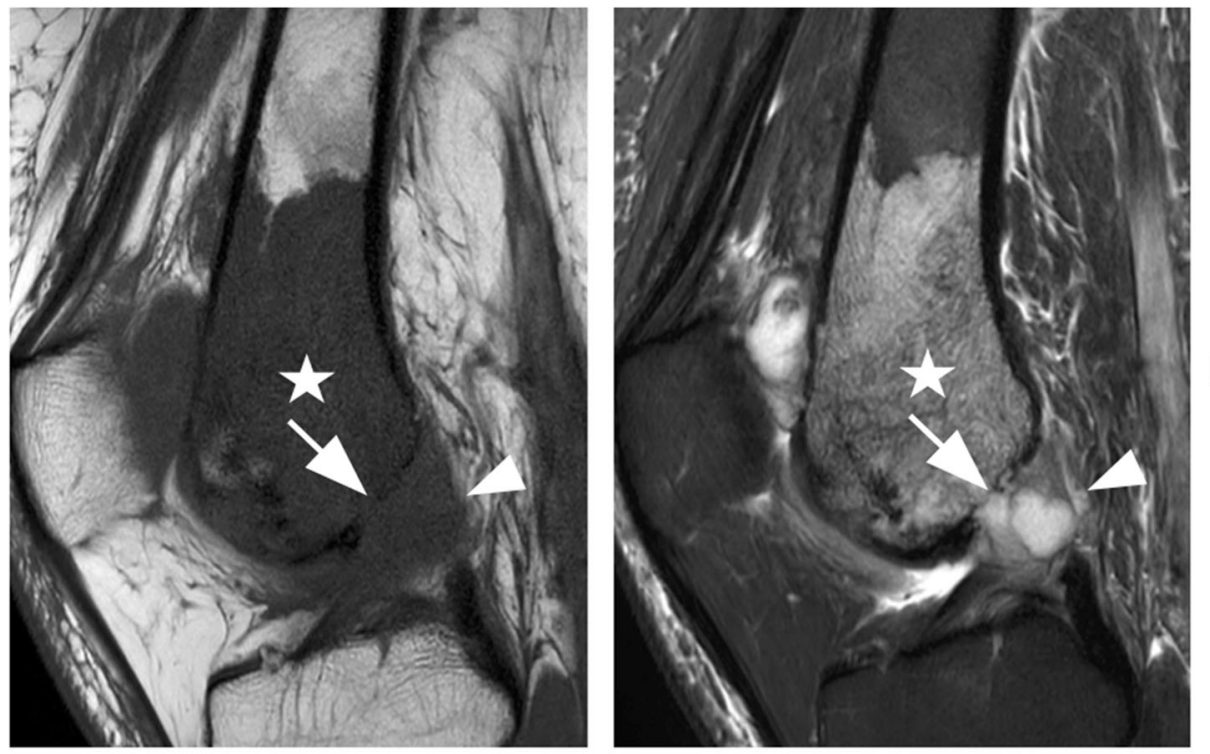

b
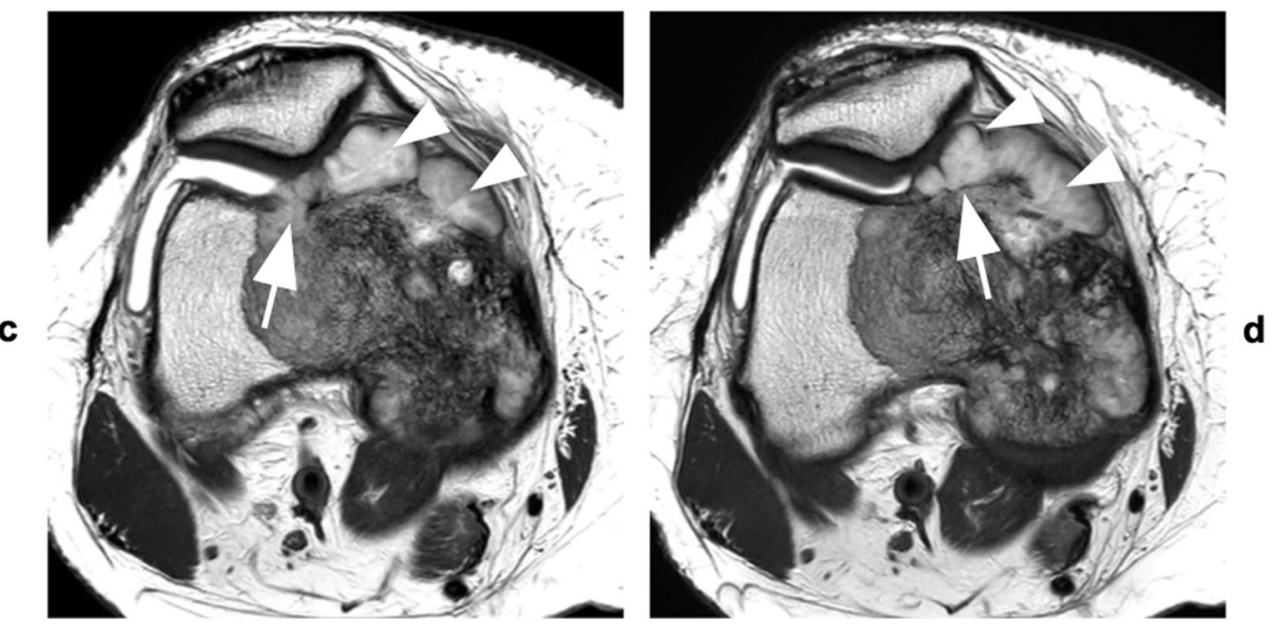

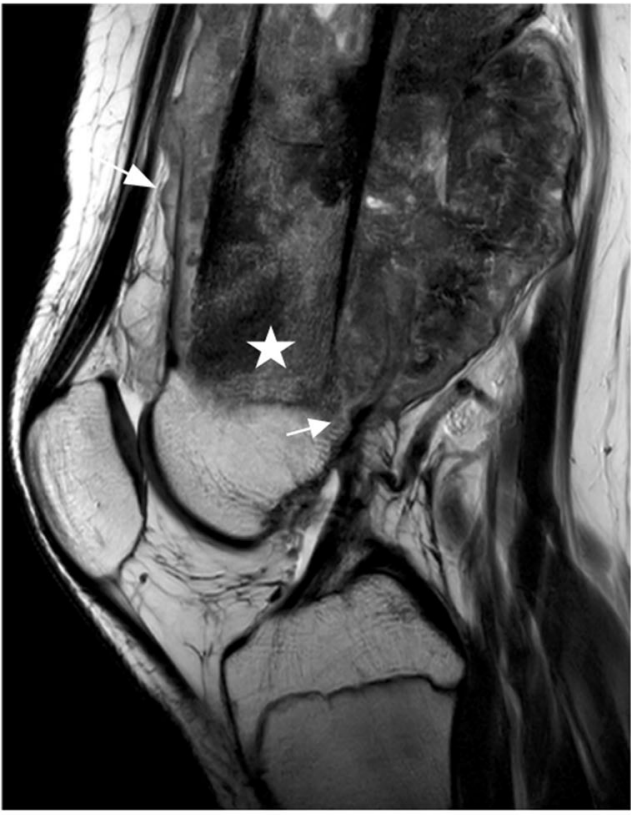

a

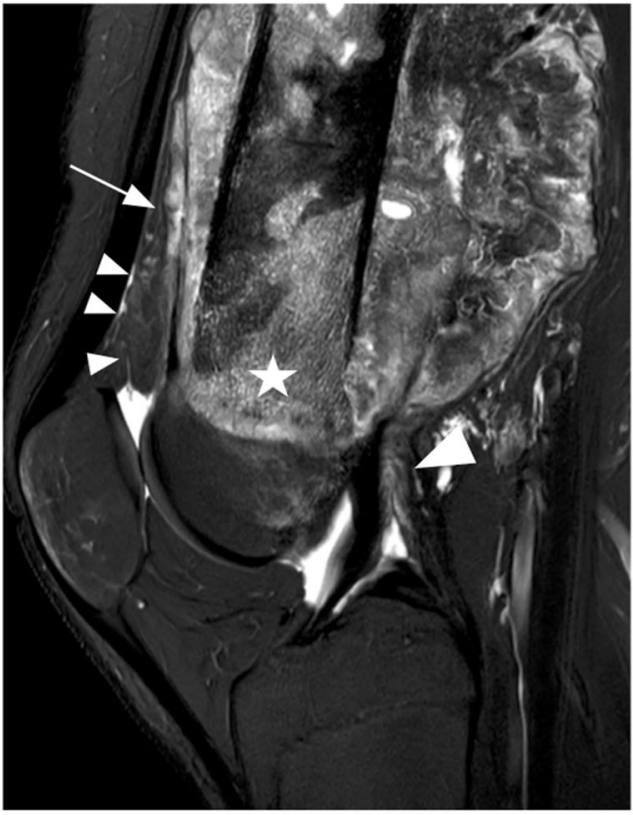

b 
across the surgical plane $[5,7,8,11,18-20]$. However, although previous studies found that the tumor diameter may precisely be measured using $\mathrm{T}_{1}$-weighted sequences, they also reported up to $50 \%$ false-positive assessments, when JI was diagnosed with MR imaging [10, 12-14, 21]. In contrast, our readers were able to confidently exclude JI in $88-100 \%$ of the true-negative cases. These differences may be explained by the improved quality of MR images compared to former years and the structured analysis of imaging signs in this study. This may also apply to the two most valuable and reproducible signs in this study - direct visualization of ITT and intraarticular destruction of bone - which were previously reported to be far less specific [12, 13, 22].

Previous studies have shown mixed results for the value of joint effusion and synovial contrast enhancement in diagnosing JI $[12,14]$. Our results indicate that these indirect signs are either nonspecific or insensitive. Such reactive changes may occur due to hyperemia, capsular irritation by the inflammatory tumor microenvironment, or mechanical stress, if the tumor is abutting the joint, but not invading it [23]. Furthermore, the absence of joint effusion showed a much lower negative predictive value for JI than in the study by Schima et al (11).

Our readers were able to detect tumor invasion of capsular insertions and to differentiate this finding from mere capsular contact or displacement, in particular with use of $\mathrm{T}_{2}$-weighted images, whereas this task was described as challenging by Schima et al, and the usefulness of $\mathrm{T}_{2}$-weighted MR sequences for diagnosing JI has been discussed controversially [12, 24]. Tumor invasion of intraarticular ligaments was a highly specific sign and showed a high PPV. For the cruciate ligaments, these results match those of previous studies, while assessment of tumor invasion of the ligamentum teres has been reported to be more difficult, most likely due to technical limitations in visualizing the ligament [13]. Notably, invasion of the hip joint via the ligamentum teres occurred most often in patients with chondrosarcomas: Out of six cases, in which invasion of the hip joint was present, chondrosarcomas accounted for four.

Similar to previous reports, epiphyseal bone marrow replacement seen on $\mathrm{T}_{1}$-weighted MR images had an excellent sensitivity and NPV, but a low specificity for the diagnosis of JI in our study [12]. Edema-like epiphyseal signal alterations were however relatively insensitive and nonspecific and may thus only be of very limited value when diagnosing JI.

The combination of highly specific direct imaging features (direct visualization of ITT, invasion of the capsular insertion, or intraarticular ligaments) led to a further increase of sensitivity (96\%), while maintaining an excellent specificity (100 $\%)$. Consequently, we propose that a combination of direct signs should be considered when diagnosing JI.

This study has limitations: most importantly, a reader bias is pertinent to this study, as due to the design of the study, readers most likely expected a high number of cases with JI, possibly influencing the diagnosis. To minimize this bias, readers were blinded to clinical and histopathological diagnoses and surgical outcome [25]. Second, the analyzed examinations were obtained in clinical routine on two MR scanners with different field strengths using pulse sequences with different in-plane resolutions. On the other hand, this also demonstrates the feasibility of assessing the discussed imaging features in routine clinical scans. Third, the statistical power of our analyses is limited by the small cohort size. It may be difficult to overcome this limitation as the incidence of joint adjacent malignant bone tumors, particularly with JI, is relatively low.

In summary, our results demonstrate the feasibility to accurately determine JI status of malignant bone tumors on pretherapeutic MR images. Direct signs, in particular visualization of intrasynovial tumor tissue and destruction of intraarticular bone, were found to be most valuable when assessing JI, owing to their excellent specificity, and were highly reproducible. In contrast to previous studies, indirect signs were found to be much less reliable. Providing this information is essential for orthopedic surgeons, as correctly diagnosing JI prior to surgery may prevent inadequate resections, postoperative complications, and local recurrence.

Supplementary Information The online version contains supplementary material available at https://doi.org/10.1007/s00330-022-08586-w.

Funding Open Access funding enabled and organized by Projekt DEAL.

\section{Declarations}

Guarantor The scientific guarantor of this publication is Prof. Dr. K. Woertler.

Conflict of interest The authors of this manuscript declare no relationships with any companies, whose products or services may be related to the subject matter of the article.

Statistics and biometry No complex statistical methods were necessary for this paper.

Informed consent Written informed consent was waived by the Institutional Review Board.

Ethical approval Institutional Review Board approval was obtained.
Methodology
- retrospective
- diagnostic or prognostic study
- performed at one institution

Open Access This article is licensed under a Creative Commons Attribution 4.0 International License, which permits use, sharing, adaptation, distribution and reproduction in any medium or format, as long as you give appropriate credit to the original author(s) and the source, provide a link to the Creative Commons licence, and indicate if changes were 
made. The images or other third party material in this article are included in the article's Creative Commons licence, unless indicated otherwise in a credit line to the material. If material is not included in the article's Creative Commons licence and your intended use is not permitted by statutory regulation or exceeds the permitted use, you will need to obtain permission directly from the copyright holder. To view a copy of this licence, visit http://creativecommons.org/licenses/by/4.0/.

\section{References}

1. Damron TA, Ward WG, Stewart A (2007) Osteosarcoma, chondrosarcoma, and Ewing's sarcoma: National Cancer Data Base Report. Clin Orthop Relat Res 459:40-47

2. Kaatsch P (2010) Epidemiology of childhood cancer. Cancer Treat Rev 36:277-285

3. Jaffe N (2009) Osteosarcoma: review of the past, impact on the future. The American experience. Cancer Treat Res 152:239-262

4. Jones KB, Ferguson PC, Lam B et al (2012) Effects of neoadjuvant chemotherapy on image-directed planning of surgical resection for distal femoral osteosarcoma. J Bone Joint Surg Am 94:1399-1405

5. Weeden S, Grimer R, Cannon S, Taminiau A, Uscinska B (2001) The effect of local recurrence on survival in resected osteosarcoma. Eur J Cancer 37:39-46

6. Simon MA, Hecht JD (1982) Invasion of joints by primary bone sarcomas in adults. Cancer 50:1649-1655

7. Tsuboyama T, Windhager R, Dock W, Bochdansky T, Yamamuro T, Kotz R (1993) Knee function after operation for malignancy of the distal femur. Quadriceps muscle mass and knee extension strength in 21 patients with hinged endoprostheses. Acta Orthop Scand 64:673-677

8. Ieguchi M, Hoshi M, Aono M et al (2014) Knee reconstruction with endoprosthesis after extra-articular and intra-articular resection of osteosarcoma. Jpn J Clin Oncol 44:812-817

9. Radiology ACo (2018) ACR Appropriateness Criteria ${ }^{\circledR}$ : Primary bone tumors,

10. Bloem JL, Taminiau AH, Eulderink F, Hermans J, Pauwels EK (1988) Radiologic staging of primary bone sarcoma: MR imaging, scintigraphy, angiography, and CT correlated with pathologic examination. Radiology 169:805-810

11. Manaster BJ (2013) Soft-tissue masses: optimal imaging protocol and reporting. AJR Am J Roentgenol 201:505-514

12. Schima W, Amann G, Stiglbauer R et al (1994) Preoperative staging of osteosarcoma: efficacy of MR imaging in detecting joint involvement. AJR Am J Roentgenol 163:1171-1175
13. Ozaki T, Putzke M, Burger H, Gosheger G, Winkelmann W, Lindner N (2002) Infiltration of sarcomas into the hip joint: comparison of CT, MRI and histologic findings in 67 cases. Acta Orthop Scand 73:220-226

14. Quan GM, Slavin JL, Schlicht SM, Smith PJ, Powell GJ, Choong PF (2005) Osteosarcoma near joints: assessment and implications. J Surg Oncol 91:159-166

15. Deng Z, Ding Y, Hao L, Zhang Q, Su Y, Niu X (2017) Marrow signal mimicking tumor on MRI T1-weighted imaging after neoadjuvant chemotherapy in extremity osteosarcomas. J Bone Oncol 6: 22-26

16. Ongolo-Zogo P, Thiesse P, Sau J et al (1999) Assessment of osteosarcoma response to neoadjuvant chemotherapy: comparative usefulness of dynamic gadolinium-enhanced spin-echo magnetic resonance imaging and technetium-99m skeletal angioscintigraphy. Eur Radiol 9:907-914

17. Holscher HC, Bloem JL, Vanel D et al (1992) Osteosarcoma: chemotherapy-induced changes at MR imaging. Radiology 182: 839-844

18. Rougraff BT, Simon MA, Kneisl JS, Greenberg DB, Mankin HJ (1994) Limb salvage compared with amputation for osteosarcoma of the distal end of the femur. A long-term oncological, functional, and quality-of-life study. J Bone Joint Surg Am 76:649-656

19. Simon M, Aschliman M, Thomas N, Mankin H (1986) Limbsalvage treatment versus amputation for osteosarcoma of the distal end of the femur. J Bone Joint Surg Am 68:1331-1337

20. Sundaram M, McLeod RA (1990) MR imaging of tumor and tumorlike lesions of bone and soft tissue. AJR Am J Roentgenol 155: 817-824

21. Pennington Z, Ahmed AK, Cottrill E, Westbroek EM, Goodwin ML, Sciubba DM (2019) Systematic review on the utility of magnetic resonance imaging for operative management and follow-up for primary sarcoma-lessons from extremity sarcomas. Ann Transl Med 7:17

22. Landis JR, Koch GG (1977) The measurement of observer agreement for categorical data. Biometrics 33:159-174

23. Mantovani A, Allavena P, Sica A, Balkwill F (2008) Cancer-related inflammation. Nature 454:436-444

24. Seeger LL, Widoff BE, Bassett LW, Rosen G, Eckardt JJ (1991) Preoperative evaluation of osteosarcoma: value of gadopentetate dimeglumine-enhanced MR imaging. AJR Am J Roentgenol 157: 347-351

25. Sica GT (2006) Bias in research studies. Radiology 238:780-789

Publisher's note Springer Nature remains neutral with regard to jurisdictional claims in published maps and institutional affiliations. 